\title{
Bildungsforschung und Bildungspolitik oder: von überdauernden Problemen der Grenzziehung Eine Replik auf Walter Herzog
}

\section{Lucien Criblez}

Der folgende Beitrag nimmt die Kritik von Walter Herzog an der Standardisierung der Schule und der Instrumentalisierung der Erziehungswissenschaft durch die Bildungspolitik in einigen wenigen zentralen Punkten auf, stellt einiges in andere $\mathrm{Zu}$ sammenhänge und präsentiert andere mögliche Lesarten der bildungspolitischen Ereignisse der letzten Jahre. Speziell wird auf die Fragen der Generierung von Steuerungswissen und auf die Frage der Autonomie der Erziehungswissenschaften eingegangen. Der dritte Teil präsentiert einige Vorschläge zur Veränderung der beklagten Situation.

«Instrumentalisierung der Erziehungswissenschaft», «Messtechnik beherrscht den Bildungsgedanken", "Theorielosigkeit der Reform» oder «technologisches Erziehungsverständnis»: Dies sind einige Schlagworte aus Walter Herzogs Kritik an der Einführung von Bildungsstandards und einigen weiteren bildungspolitischen Reformprojekten in der Schweiz der letzten Jahre. Die Schlagworte verdeutlichen, dass die Kritik nicht einfach dem wissenschaftlich rationalen Abwägen von Argument und Gegenargument verpflichtet ist, sondern ihren Wert vor allem darin hat, eine Diskussion anzuregen. Diesem Zweck dient denn auch die zuspitzende und polarisierende Rhetorik, die einerseits die Argumentation verdeutlicht, andererseits an einigen Stellen auch in Polemik umzuschlagen droht. Der Zweck der Diskussionsanregung ist mit den verschiedenen Reaktionen in diesem Heft jedenfalls bereits erfüllt. Die Diskussion sollte jedoch nach dem polarisierenden Input differenzierter weitergeführt werden.

Der Beitrag von Walter Herzog kann meines Erachtens aus unterschiedlichen Perspektiven heraus gelesen werden und die Bewertung der Argumentation verändert sich, wenn man ihn mehr als einmal liest. Beim ersten Lesen ist man als (reform-)pädagogischer «Gutmensch» geneigt, in vielerlei Hinsicht zuzustimmen - auch wenn der Vergleich der arbeitsteiligen und technologischen Fertigung des Maschinengewehrs mit der Bildung von Kindern und Jugendlichen auch auf den ersten Blick etwas weit hergeholt und als Vergleich von nicht-Vergleichbarem erscheint. Als bildungspolitisch Interessierter teilt man vielleicht ei- 
nen Teil der Kritik an den gegenwärtig vorherrschenden Tendenzen der Bildungspolitik $^{1}$ - auch wenn sich sofort Zweifel über Walter Herzogs implizite normative Prämissen, was und wie Bildungspolitik zu sein hat, und wie deren Verhältnis zur Erziehungswissenschaft sein soll, einstellen. Als Wissenschaftler und Universitätsangehöriger ist man jedenfalls geneigt, die Autonomieforderungen für die Wissenschaft zu unterstützen - auch wenn sich der Glaube an eine «reine» Wissenschaft, zumal eine «reine» Erziehungswissenschaft als Teil der Sozialwissenschaften, schon längst als Jungakademiker-Illusion erwiesen hat. Als Erziehungswissenschaftler teilt man fast mit Sicherheit die Kritik am zu geringen Einfluss der eigenen Disziplin und an der Tendenz, dass pädagogische Themen von andern Disziplinen, insbesondere der Betriebswirtschaft und den Staatsund Verwaltungswissenschaften, vereinnahmt werden - auch wenn sich die Bildungsforschung in der Schweiz explizit nie auf die Erziehungswissenschaften reduzieren lassen wollte (Criblez, 1999a; Gretler, 1982) und zum Beispiel an der Universität Genf, wo die Disziplin in der Schweiz am stärksten ausgebildet und am differenziertesten ist, die «Sciences de l'éducation» (im Plural) seit längerem als interdisziplinäres Themenfeld, in dem Fragen und Probleme aus unterschiedlicher disziplinärer Sicht bearbeitet werden, verstanden werden (Hofstetter, 2007, S. 105-134).

Liest man den Beitrag ein zweites, drittes oder viertes Mal, werden Widersprüche in der Argumentation deutlich und es stellen sich dem historischen Bildungsforscher einige Fragen, u.a. zu den historischen Prämissen verschiedener Argumente, zur allgemeinen "Verfallsthese» der Erziehungswissenschaft oder zum Revival der Forderung nach einer Bildungstheorie als Grundlage der Bildungspolitik, vorgetragen von einem Pädagogischen Psychologen, der sich verschiedentlich für eine empirische Wende in der Erziehungswissenschaft eingesetzt hat. Jemandem, der sich um die Erforschung der Bildungspolitik in der Schweiz bemüht, fällt bei vertiefter Lektüre auch auf, dass ein Teil der Kritik an der Instrumentalisierung der Erziehungswissenschaft auf einem Verständnis von Bildungspolitik, Bildungsforschung und deren Verhältnis zueinander beruht, das erstens ahistorisch ist und zweitens in Teilbereichen von Prämissen ausgeht, die man nicht zwingend teilen muss. Letztlich ist man erstaunt, dass der Beitrag neben der Kritik an keiner Stelle fragt, weshalb das so ist, wie es ist (wenn denn die kritischen Analysen richtig sind) und dass mit Ausnahme des Hinweises auf die fehlende Bildungstheorie kein einziger Vorschlag entwickelt wird, wie die als misslich erachtete Situation verändert werden könnte.

Meine Replik kann nur einige wenige Aspekte thematisieren. Ich konzentriere mich auf drei Punkte: Zunächst gehe ich auf Fragen der Steuerung des Bildungssystems ein, weil sich die Kritik von Walter Herzog massgeblich an der Einführung von Bildungsstandards entzündet. Ich stelle den Diskurs um Bildungsstandards in andere Kontexte als Walter Herzog dies tut, und präsentiere vor diesem Hintergrund eine andere Lesart der Ereignisse und Entwicklungen als Walter Herzog. Zweitens gehe ich auf das Verhältnis von Bildungspolitik und 
Bildungsforschung und die von Walter Herzog vertretene These des Autonomieverlustes der Erziehungswissenschaft ein. Vor diesem Hintergrund unterbreite ich drittens einige Vorschläge, die vielleicht in der Lage sind, die Diskussion produktiv fortzusetzen.

\section{Steuerung des Bildungssystems}

Der Diskurs um eine neue Steuerung des Bildungssystems beginnt nicht erst mit dem Projekt HarmoS der Schweizerischen Konferenz der kantonalen Erziehungsdirektoren (EDK) oder dem Projekt Bildungsmonitoring von Bund und Kantonen, sondern mit der Diskussion um die Neue Verwaltungsführung oder New Public Management im Bildungsbereich in der Mitte der 1990er-Jahre (vgl. u.a. Criblez, 1996; Dubs, 1996). Diese Diskussion wiederum ist einerseits nicht einfach eine genuin pädagogische Diskussion, sondern vor dem Hintergrund eines grundlegenden Vertrauensverlustes einer breiten Öffentlichkeit gegenüber den Leistungen der öffentlichen Hand, also der Art und Weise, wie der Staat seine Aufgaben wahrnimmt, zu verstehen (Schedler, 1995). Gleichzeitig zeichneten sich mit den wirtschaftlichen Einbrüchen zu Beginn der 1990erJahre immer deutlicher Finanzierungsprobleme der öffentlichen Hand ab, so dass Sparprogramme nun auch den Bildungsbereich tangierten. Andererseits ist vor dem Hintergrund einer Kritik an der traditionellen Schulreform "von oben» (programmatisch: Rolff, 1984) eine Reform «von unten» mit Begriffen wie «Schulentwicklung», «Schulautonomie» und «Schulqualität» eingefordert worden. Diese Forderungen - insbesondere diejenige nach mehr Autonomie - fanden in Schulkreisen breite Unterstützung, auch wenn von Anfang an die Kehrseite grösserer Autonomie, nämlich die Verstärkung der Rechenschaftspflicht (durch vergleichende Leistungsmessung, Leistungslohn für das Personal sowie externe Schulevaluation) abgelehnt wurde. Pointiert formuliert: Die international geführte Schulentwicklungs- und Schulqualitätsdiskussion wurde insbesondere in Kreisen der Lehrerschaft, aber auch von der Bildungspolitik selektiv rezipiert.

Dies verweist aber auf einen dritten Zusammenhang, der für das Verständnis der Entwicklungen zentral ist: Das schweizerische Bildungswesen begann sich seit Ende der 1980er-Jahre nach aussen zu öffnen (Criblez, 2007). Erstmals unterzog sich die schweizerische Bildungspolitik einer OECD-Review (EDK, 1990) und die schweizerische Bildungsstatistik stellte sich auf international vergleichbare Bildungsindikatoren ein, bei denen erstmals die Output- bzw. Outcomes-Indikatoren eine wichtige Rolle spielten. Zudem beschlossen die Kantone, sich an den PISA-Studien zu beteiligen. Diese internationale Öffnung zeigte nun aber mit grosser Deutlichkeit, dass die Bildungspolitik in der Schweiz seit dem 19. Jahrhundert weitgehend kantonale Bildungspolitik gewesen und dass gar nie eine nationale Bildungspolitik entstanden war ${ }^{2}$ (Criblez, 2007). 
Die je kantonalen Bildungspolitiken standen in den 1990er-Jahren also unter mehrfachem Reformdruck: Erstens sollte die Qualität von Schule nachweisbar erhöht werden, zweitens sollte den Schulen mehr Freiraum zugebilligt werden, drittens sollten Mechanismen der neuen Verwaltungsführung auch auf den Bildungsbereich übertragen werden, viertens musste auch der Bildungsbereich zu den Einsparungen der öffentlichen Hand beitragen und fünftens mussten sich die Kantone insbesondere wegen der Personenfreizügigkeit und Diplomanerkennung (Criblez, in Druck [2008a]) auf internationale Vorgaben einstellen, was eine bessere Koordination innerhalb der Schweiz voraussetzte. Dass in dieser Situation noch das alte "Schreckgespenst» des eidgenössischen Schulvogtes abzuwehren war, der sich in einer Parlamentarischen Initiative ankündigte (Bundesrat, 2005; Kommission NR, 2005), die letztlich zu Änderung der Bildungsverfassung im Mai 2006 führte, liess die Kantone zusammenrücken und die EDK ein Reformprogramm formulieren (Criblez, in Druck [2008b]).

Die beiden Projekte HarmoS und Bildungsmonitoring sind - so gesehen - als Reaktion vor allem der EDK (beim Bildungsmonitoring auch des Bundes) auf diese verschiedenen Herausforderungen zu verstehen, das HarmoS-Projekt (das zwei Teile umfasst, einen politischen mit dem Ziel der Etablierung eines neuen multilateralen Staatsvertrages, und einen inhaltlichen mit dem Ziel der Definition von Bildungsstandards; EDK, 2004; Maradan \& Mangold, 2005) insbesondere auch als Projekt der Selbstverpflichtung der Kantone auf Koordination, nicht zuletzt um weiterreichende Bundeslösungen zu verhindern - was mit der neuen Bildungsverfassung auch erreicht wurde: Sie beschneidet die Regelungskompetenzen der Kantone im Bildungswesen in keinem Bereich wirklich, unterstützt sie aber in den Koordinationsbemühungen.

Historisch gesehen hat diese Entwicklung durchaus eine bestimmte Logik: Die Standards zur Steuerung des Bildungssystems setzten seit dem 19. Jahrhundert die Kantone. Sie haben Standardisierungen in ihrem Hoheitsgebiet spätestens seit den 1830er-Jahren immer mit notwendigen Qualitätssteigerungen und Innovationen begründet: Die Einführung von Normalmethode und Normalschulen (Ecole normale; also der modernen Lehrerinnen- und Lehrerbildung) hatten nichts anderes bezweckt. Kantonale Schulgesetze und Lehrpläne waren immer schon (nicht nur, aber auch) kantonale Steuerungsinstrumente, um Gemeinden und Schulen auf minimale Standards zu verpflichten (Criblez, 1999b; Rhyn, 1998, S. 163-187). Es ging dabei durchaus nicht nur um Input- oder opportunity-to-learn-Standards, sondern die Pädagogischen Rekrutenprüfungen (Crotti, in Druck [2008]; Lustenberger, 1996), die in verschiedenen Kantonen verbreiteten Schulendprüfungen, die so genannten «Examen» jeweils am Ende des Schuljahres oder auch die Besuche des Schulinspektors hatten den Charakter der Ergebnisüberprüfung.

So gesehen sind weder Standardisierungen noch Ergebnisstandards etwas völlig Neues in der Schulgeschichte. Die Steuerungsversuche hatten allerdings historisch sehr unterschiedliche Formen angenommen. Insbesondere von den Pä- 
dagogischen Rekrutenprüfungen können wir annehmen, dass sie heutigen wissenschaftlichen Standards (etwa der Validität oder der Reliabilität) nicht standgehalten hätten. Trotzdem haben sie wesentlich zur Verbesserung des Schulwesens in vielen Kantonen beigetragen, weil den Resultaten eine bestimmte öffentliche Aufmerksamkeit zukam. In diesem Sinne müsste auch für die neuen Steuerungsinstrumente zumindest zwischen ihrer beabsichtigten Wirkung und ihrer tatsächlichen Wirkung, mit einem besonderen Augenmerk auf unerwartete Effekte (Merton, 1936), unterschieden werden. Für die Beurteilung von Wirkungen der neuesten Steuerungsversuche ist es aber bislang zu früh. Immerhin hat das HarmoS-Projekt als unerwarteten Effekt die Etablierung von empirischer Forschung in den Fachdidaktiken in der Schweiz stark befördert, wie die in den letzten drei Jahren vom Schweizerischen Nationalfonds zur Förderung der wissenschaftlichen Forschung bewilligten Forschungsprojekte in der anwendungsorientierten Projektförderung DORE (Do Research) zeigen.

Verändert hat sich allerdings die politische Ebene der Steuerungsversuche: Nicht mehr die je einzelnen Kantone etablieren Steuerungsmechanismen, aber auch nicht der Zentralstaat (Bund), sondern die EDK als Organ aller Bildungsbzw. Erziehungsdirektoren und -direktorinnen. Auch diese Art von Steuerung ist nicht ganz neu, sie wurde im Bildungsbereich erstmals 1970 mit dem Schulkonkordat (EDK, 1970, S. 91-94)) eingeführt, zunächst allerdings mit wechselndem Erfolg (Arnet, 2000). Mit verschiedenen interkantonalen Vereinbarungen und dem HarmoS-Konkordat hat die Interkantonalisierung seit den 1990er-Jahren aber neue Dimensionen erreicht. Diese Steuerung auf interkantonaler Ebene, eine Art Interkantonalisierung zur Verhinderung der Nationalisierung, hat ihre Begründung u.a. in der Internationalisierung (Caruso \& Tenorth, 2002; Osterwalder \& Weber, 2004) der Bildungspolitik, und man kann aus staatsrechtlichen und staatspolitischen Gründen durchaus fragen, ob das von der EDK etablierte System der multilateralen Staatsverträge tauglich ist, um die Herausforderungen der Bildungspolitik im 21. Jahrhundert effizient und effektiv zu bearbeiten (Criblez, in Druck [2008b]). Dass aber die kleinräumige Bildungspolitik im traditionellen schweizerischen Bildungsföderalismus angesichts der "Weltgesellschaft» (Stichweh, 2000) inzwischen mehr Probleme schafft als löst, dürfte zumindest der Schweizer Souverän bei der Abstimmung über die neue Bildungsverfassung im Mai 2006 verstanden haben. Es geht also bei der Einführung von Bildungsstandards nicht um die Reaktivierung eines «fragwürdigen Nationendenkens» (Walter Herzog), sondern um die Neudefinition von Steuerungsprozessen auf überkantonaler Ebene, die bei weitem noch nicht etabliert sind (detaillierter: Criblez, in Druck [2008b]).

Die Projekte HarmoS und Bildungsmonitoring stehen damit wie die neue Bildungsverfassung einerseits für neue Versuche der Steuerung des Bildungswesens, insbesondere aber vor allem für die Steuerung aufeiner anderen als der kantonalen politischen Ebene. Dass dies alte Steuerungsmechanismen und die bisherige Verteilung von Regelungskompetenzen im schweizerischen Bildungssystem 
in Frage stellt, versteht sich von selbst. Dass die Instrumente eher technologisch konzipiert sind, ist eine sicher berechtigte Kritik, die auch an anderer Stelle bereits vorgetragen wurde (detaillierter: Lehmann, Criblez, Guldimann, Fuchs \& Périsset Bagnoud, 2007, S. 113ff.). Aber es geht um eher technologische Vorstellungen zur Steuerung des Bildungssystems, nicht um Bildungs- oder Erziehungstechnologie, wie Walter Herzog unterstellt. Dass in der bildungspolitischen Alltagspraxis einige solcher zentraler Unterscheidungen nicht durchgehalten werden, kann man beklagen, aber nicht den bildungspolitischen Steuerungsprojekten selbst vorwerfen. Tatsächlich stellen sich die Fragen der Unterscheidung unterschiedlicher Ebenen und Messinstrumente dann noch ein bisschen komplizierter, als Walter Herzog sie darstellt. Letztlich geht es um die Frage, wie in Zukunft Leistungen auf der Ebene der einzelnen Schülerinnen und Schüler, der einzelnen Klassen, der einzelnen Schulen, der Schulgemeinden, der kantonalen Schulsysteme und des Schulsystems Schweiz im internationalen Vergleich sinnvoll bewertet werden können, und welche Evaluationsinstrumente auf welcher Ebene wissenschaftlich legitimiert eingesetzt werden können. Dass ein "grobmaschiges» Instrument zur Evaluation des schweizerischen Bildungssystems nicht zu Selektionszwecken für einzelne Schülerinnen und Schüler eingesetzt werden kann, dass zwischen Einzelschule und Schulsystem unterschieden werden muss und dass die Funktionen von Förderung und Selektion zu trennen sind und unterschiedliche Diagnostikinstrumente benötigen, ist der Wissenschaft klar, nicht immer und überall aber der Bildungspolitik.

\section{Bildungspolitik und Bildungsforschung/Erzie- hungswissenschaft}

Für das Folgende will ich von einer einfachen Prämisse ausgehen: Es ist die genuine Aufgabe der Bildungspolitik, das Bildungswesen zu steuern (auch wenn z.B. die Finanzpolitik ebenfalls an der Steuerung beteiligt ist). Die Bildungspolitik muss im Sinne der Steuerung die Rahmenbedingungen für Bildungsinstitutionen auf allen Stufen des Bildungssystems festlegen - zumindest wenn Bildung zu den Kernaufgaben des Staates gehören soll (was gerade durch die neue Bildungsverfassung noch einmal bestärkt worden ist). Bildungspolitik hat also letztlich eine normative Aufgabe. Ist dies als Prämisse akzeptiert, stellt sich die Frage, wie die Bildungspolitik ihre im Kern normative Aufgabe am besten wahrnehmen kann. Seit den späten 1960er-Jahren hat sich das Ideal einer «rationalen Bildungspolitik» (Widmaier, 1966; Widmer, 1976) durchgesetzt, einer Bildungspolitik also, die auf der Grundlage wissenschaftlicher Forschung operiert. Was damals als Bildungsplanung mit den zeitgenössischen Planungshoffnungen («Die Zukunft ist errechenbar ...»; Stettler, 1994) konzipiert wurde, führte mehr oder weniger direkt zu kantonalen Planungsstellen im Bildungsbereich, später als Pädagogische Arbeitsstellen bezeichnet (Bain, Brun, Hexel \& Weiss, 2001), die 
wiederum einen Teil ihrer Aufgaben anfangs dieses Jahrzehnts an die neu gegründeten Pädagogischen Hochschulen abgaben.

Ich gehe davon aus, dass der Grundsatz, dass sich bildungspolitische Steuerung in einer versozialwissenschaftlichten Gesellschaft auf wissenschaftliche Forschungsergebnisse abstützen soll, nicht bestritten ist ${ }^{3}$. Denn Politik bzw. politische Macht beruht heute in demokratischen Staaten auf doppelter Legitimation: auf der Legitimation der Machtdelegation durch Wahl (Criblez, 1999b) und auf der Legitimation politischer Entscheide durch deren Fundierung auf gesicherten und wissenschaftlich konsensfähigen Erkenntnissen (Weingart, 2006, S. 36). Akzeptiert man dies (ein Rückfall hinter diesen Stand wäre aus meiner Sicht für Bildungspolitik und Bildungsforschung äusserst schädlich), kann man nicht mehr das Bemühen der Politik beklagen, ihre Entscheide auf wissenschaftliche Erkenntnisse (die in der Regel durch Expertisen eingeholt werden) abzustützen. Es lässt sich dann kritisieren, dass sich die Bildungspolitik zu wenig theorieorientiert auf Zahlen, Daten und Fakten abstützt, oder dass die Wissenschaft durch das Auftragsverhältnis in Abhängigkeiten gerät, die eine objektive Expertise verhindern.

Zumindest auf Seiten der Bildungsforschung sollte das Verhältnis zwischen Bildungsforschung und Bildungspolitik auf der Grundlage des sozialwissenschaftlichen Erkenntnisstandes definiert werden: Sowohl die Systemtheorie als auch die sozialwissenschaftliche Verwendungsforschung der 1980er-Jahre haben auf die strukturellen Differenzen zwischen Wissenschaft und Politik hingewiesen: Systemtheoretisch gesehen sind die Erziehungswissenschaft als Teil des Wissenschaftssystems und die Schule als Teil des Bildungssystems Teil der Umwelt des politischen Systems. Alle Teilsysteme folgen je eigenen Rationalitäten und Funktionslogiken, ein Austausch ist nur über Kommunikation möglich (Luhmann, 1987). Würde die Wissenschaft die Funktionslogik der Politik übernehmen, würde sie selber zur Politik (Kussau \& Oertel, 2001). Der Konflikt zwischen Wissenschaft und Politik ist "unauflöslich und prinzipieller Natur» (Weingart, 2006, S. 36), was Folgen für die Politikberatung durch Wissenschaft hat. Vor diesem Hintergrund könnte man argumentieren, dass die Theorieproduktion eine Funktion der Wissenschaft, nicht der Politik ist. Der Vorwurf der Theorielosigkeit, den Walter Herzog vorbringt, fällt dann auf die Wissenschaft zurück, die sich bislang tatsächlich wenig mit der Funktionsweise von Steuerungsprozessen in Bildungssystemen auseinander gesetzt hat. So ist denn nicht erstaunlich, dass den bildungspolitischen Reformprojekten relativ einfache Modelle der Steuerung zugrunde liegen (SKBF, 2006, S. 7; kritisch: Lehmann et al. 2007, S. 113f.).

Aus verschiedenen Studien der sozialwissenschaftlichen Verwendungsforschung wissen wir zudem, dass die Bildungspolitik Resultate von sozial- und erziehungswissenschaftlicher Forschung nicht nach den Regeln der Wissenschaft, sondern nach den Regeln der Bildungspolitik rezipiert. Jedenfalls existiert kein «Rationalitätskontinuum» von der Wissenschaftstheorie über die Wissenschaft 
zu wissenschaftlich aufgeklärtem Handeln und Entscheiden (Drerup, 1989, S. 146). Anders als die «naive Verwendungsforschung» (Beck \& Bonss, 1984) angenommen hat, ist die Bildungspolitik nicht passive Konsumentin von Forschung, sondern aktive Re-Produzentin. Der Umgang mit wissenschaftlichen Informationen ist von Diskontinuitäten geprägt (Drerup, 1989, S. 155). Das einfache Regelkreismodell zwischen Bildungsforschung und Bildungspolitik/verwaltung, wie es dem Bildungsmonitoring in der Schweiz zugrunde liegt (SKBF, 2006, S. 7), ist schon aus diesen Gründen (aber auch aus anderen; vgl. Lehmann et al., 2007, S. 113f.) zu wenig differenziert.

Allerdings deutet die sozialwissenschaftliche Verwendungsforschung auf ein weiteres Problem hin: Sozialwissenschaftliche Forschungsergebnisse können von der Bildungspolitik - wie auch immer sie rezipiert und verwendet werden - nur rezipiert werden, wenn sie überhaupt generiert worden sind. Auch in dieser Hinsicht fällt die Kritik eigentlich auf die Wissenschaft zurück: Die Produktion von Erkenntnissen über Bildungsinstitutionen und Bildungsprozesse gehört zum genuinen Auftrag der Erziehungswissenschaften. Die Disziplin muss nicht auf Aufträge von Seiten der Bildungsforschung warten. In diese Richtung zielte wohl die (in dieser Hinsicht aus meiner Sicht berechtigte) Kritik an der mangelnden Produktion von Steuerungswissen durch die Schweizer Bildungsforschung des OECD-Expertengremiums über die Bildungsforschung - auch wenn die Fragestellung der Expertise zu eingeschränkt war und einiges im Bericht mit einer besseren und ausgewogeneren Informationsbasis anders beurteilt hätte werden können (OECD/CERI, 2007b; SKBF \& CORECHED, 2006).

Lässt sich also die Erziehungswissenschaft instrumentalisieren, wenn sie an der Beschaffung von Grundlagen für die Definition von Bildungsstandards mitarbeitet oder Daten generiert und aufbereitet, die der Bildungspolitik als Grundlage für Steuerungsentscheide dienen? Verliert die Erziehungswissenschaft dadurch ihre «Unabhängigkeit» und wird sie «ihrer kritischen Funktion» beschnitten? Und welche «Werte» stehen «auf dem Spiel, deren Verlust nicht widerstandslos hingenommen werden kann» (Walter Herzog)? Herzogs Besorgnis um eine autonome Erziehungswissenschaft ist zwar ehrenhaft, die Geschichte der Erziehungswissenschaft in der Schweiz (Hofstetter \& Schneuwly, 2007), ja auch die Geschichte der Sozialwissenschaften (Honegger, Jost, Burren \& Jurt, 2007) zeigen aber, dass die sozialwissenschaftlichen Disziplinen sich kaum nur nach innen legitimieren können, sondern dass sie auf Aussenlegitimierung angewiesen sind. Zwar vermögen Prozesse der Institutionalisierung (Etablierung an Universitäten, Gründung von Gesellschaften und Zeitschriften usw.) einer Disziplin mehr Autonomie zu verschaffen, so dass sie sich weniger gegenüber ausserwissenschaftlichen Instanzen zu legitimieren braucht. Aber auch die Physik gerät nach einem schweren AKW-Unfall in Legitimationsprobleme. Bourdieu (1998, S. 17) hat deshalb die Idee einer vollständigen Autonomie von Wissenschaft als unzulässige Projektion der Wissenschaftstheorie auf die wissenschaftliche Praxis bezeichnet. 
Trotzdem ist Herzog natürlich zuzustimmen, dass die Erziehungswissenschaft zu wenig autonom ist. Sie teilt dieses Problem mit den andern Sozialwissenschaften, die insbesondere in der Schweiz bis weit ins 20. Jahrhundert eng an die Politik angebunden blieben, und deren Autonomisierung dadurch gebremst und erschwert wurde. Denn die enge Verbindung der Sozialwissenschaften mit den politischen Eliten habe dazu geführt, "dass die wissenschaftlichen Strategien auf kurzfristige gesellschaftliche Bedürfnisse und kaum auf langfristige wissenschaftliche Zielvorstellungen ausgerichtet wurden» (Honegger et al., 2007, S. 12). Dies trifft für die Bildungsforschung in der Schweiz in besonderer Weise zu. Zwei Beispiele mögen dies dokumentieren: Die Schweizerische Gesellschaft für Bildungsforschung hat seit ihrer Gründung einen Teil ihrer Vorstandsmitglieder aus der Bildungsverwaltung, aus Pädagogischen Arbeitsstellen oder andern politiknahen Institutionen rekrutiert. Der Name ihrer Zeitschrift «Bildungsforschung und Bildungspraxis», in deren Redaktion Walter Herzog lange Jahre Mitglied war, stand für ein Programm, das eher für die ,Kontaminierung' der reinen Wissenschaft durch Praxis und Politik als für die Autonomie der Wissenschaft stand. Und: Wegen des geringen Ausbaus und der geringen Differenzierung der Disziplin Erziehungswissenschaft an den Universitäten wurde die Bildungsforschung von anderen Institutionen und Disziplinen wahrscheinlich ebenso stark geprägt wie durch die akademische Erziehungswissenschaft.

Zwar ist die Anzahl der universitären Professuren in den Erziehungswissenschaften in den letzten Jahren angestiegen, aber die Situation hat sich vor allem durch den Aufbau von anwendungsorientierter Forschung in der Lehrerinnenund Lehrerbildung grundlegend verändert. Allerdings ist dadurch eben nicht vor allem die freie Forschung, sondern die orientierte Forschung und Entwicklung gefördert worden. Dies hat verschiedene Gründe: Weil die Einheit von Forschung und Lehre für die Lehrerinnen- und Lehrerbildung nicht wie an Universitäten gilt und nach wie vor kaum ein wissenschaftlicher Mittelbau existiert, sind die Pädagogischen Hochschulen sehr viel stärker auf Drittmittelakquisition angewiesen als Universitätsinstitute. Zweitens sind die Pädagogischen Hochschulen per definitionem auf anwendungsorientierte Forschung verpflichtet und drittens haben sie von den Kantonen zumindest teilweise Aufgaben der ehemaligen Bildungsplanungsstellen bzw. Pädagogischen Arbeitsstellen übernommen. Ein wesentlicher Teil der in der ersten Forschungslandkarte der Lehrerinnenund Lehrerbildung verzeichneten Forschungs- und Entwicklungsprojekte (Vogel, 2006) dürfte deshalb so genannt orientierte Forschung und Entwicklung bzw. Dienstleistung sein. Die Disziplin ist also wahrscheinlich institutionell gespalten, in einen autonomeren Teil der Erziehungswissenschaften an den Universitäten und einen weniger autonomen Teil an den Pädagogischen Hochschulen, der stärker auf Aussenlegitimierung angewiesen ist. Das kann man im Hinblick auf Autonomie der Disziplin beklagen, aber nicht den Pädagogischen Hochschulen zum Vorwurf machen. 


\section{Einige Perspektiven}

Die Wissenschaft kann sich nicht anmassen, der Bildungspolitik und -verwaltung vorzuschreiben, wie sie mit wissenschaftlichen Erkenntnissen umzugehen hat. Aber sie kann mit wissenschaftlichen Methoden die Verwendung von wissenschaftlichen Erkenntnissen in Politik und Verwaltung untersuchen und die Resultate öffentlich zur Diskussion stellen - ein in der Schweiz unbearbeitetes Forschungsfeld der Bildungsforschung. Bildungspolitik und Bildungsverwaltung haben das Recht (und die staatspolitische Pflicht!), Daten und Erkenntnisse zu generieren oder generieren zu lassen, mit denen sie in die Lage versetzt werden, Bildungspolitik auf bessere und transparentere Grundlagen abzustützen als den jeweils aktuellen (partei-)politischen Mainstream. Die kleinen und mittleren, zunehmend aber auch die grossen Kantone sind nicht (mehr) in der Lage, diese rationalen Grundlagen der Bildungspolitik für alle Bildungsbereiche permanent zu beschaffen.

Letztlich hat auch diese Erkenntnis der wissenschaftlichen Überforderung im kleinräumigen Bildungsföderalismus dazu geführt, dass die wichtigsten Projekte zur Grundlagenbeschaffung im Bildungsbereich heute auf interkantonaler, sprachregionaler oder nationaler Ebene angesiedelt sind. Sofern die Aufträge von Seiten der Bildungsforschung nach den Regeln der Kunst entgegengenommen und realisiert werden, kann die Bildungsforschung von diesen Aufträgen profitieren, allerdings nur solange die Auftragsforschung nicht zur dominierenden Form der Forschung wird, das heisst: so lange die freie Forschung über genügend Potential verfügt, die Erkenntnisproduktion unabhängig vom - und wenn gerechtfertigt auch gegen den - politischen Mainstream zu gewährleisten. Über kritische Grenzen wäre zu diskutieren und es könnte sein, dass diese kritischen Grenzen an einigen Pädagogischen Hochschulen überschritten sind.

Zusammenfassend wäre mein Plädoyer als Reaktion auf Walter Herzogs Artikel also: Die Erziehungswissenschaft in der Schweiz sollte sich nicht in «Bildungspolitikschelte» üben, sondern insbesondere die eigene Disziplin stärken, damit sie das Verhältnis zwischen Bildungspolitik und Bildungsverwaltung aus der Position einer starken und autonomen Disziplin heraus besser mitdefinieren kann. Dazu könnte einiges unternommen werden. Ich beschränke mich auf sechs einigermassen konkrete Vorschläge:

- Um die Autonomie der Disziplin zu stärken, müsste die Selbstorganisation der Disziplin in Richtung einer stärkeren Institutionalisierung verändert werden. Die Schweizerische Gesellschaft für Bildungsforschung und die Schweizerische Gesellschaft für Lehrerinnen- und Lehrerbildung müssten fusionieren, um in einer gemeinsamen Schweizerischen Gesellschaft der Erziehungswissenschaften den Grad von Institutionalisierung zu erreichen, der eine höhere Autonomie gegenüber der Bildungspolitik absichert. 
- Die wissenschaftliche Disziplin sollte sich - in Eigenverantwortung und aus eigener Initiative - ein Set von Regeln für die Auftragsforschung geben, auf die sich die Mitglieder der Disziplin freiwillig verpflichten, eine Art Codex der erziehungswissenschaftlichen Auftragsforschung.

- Fragen der disziplinären Autonomie, der Disziplinengeschichte, des Verhältnisses von Bildungspolitik und Bildungsforschung sowie der Auftragsforschung sind zu zwingenden Inhalten der Ausbildung des wissenschaftlichen Nachwuchses zu machen. Dazu muss die Nachwuchsförderung innerhalb der Disziplin aber zunächst besser institutionalisiert werden.

- Die Diskussionen zwischen Bildungsforschung und Bildungspolitik über deren Verhältnis, deren gegenseitige Erwartungen und die Frage der Forschung im Auftrag von Bildungspolitik und -verwaltung, die in Ansätzen aufgrund der OECD-Review begonnen worden sind, müssen fortgesetzt werden. Die Erziehungswissenschaft ist auch in Zukunft zumindest teilweise auf Aussenlegitimation angewiesen. Die gegenseitigen Erwartungen sollten aber besser geklärt werden.

- Die Bildungsforschung steht vor der Herausforderung, Fragen der bildungspolitischen Steuerung wissenschaftlich zu bearbeiten. Das Themenfeld, das in der angelsächsischen oder französischen Diskussionstradition einen ganz anderen Stellenwert hat als in der schweizerischen, sollte von der Disziplin Erziehungswissenschaft bearbeitet werden und nicht andern Disziplinen überlassen werden.

- Für die Bildungsforschung wäre es hilfreich, wenn Bildungspolitik und -verwaltung klarere und eingegrenztere Fragen und Aufträge stellen würden und sich der Unterschiede zwischen Forschung und Politik ebenfalls stärker bewusst wären. Dies kann von Seiten der Wissenschaft aber nicht mehr als gewünscht und angemahnt werden.

Dies sind nur einige wenige konkrete Vorschläge, die vielleicht in der Lage sind, die Diskussion weiter anzuregen. Die Vorstellung aber, dass eine allgemeine Bildungstheorie in der Lage wäre, die Fragestellungen und Projekte der Bildungsreformen quasi deduktiv zu leiten, gehört meines Erachtens eher zu den nationalstaatlichen und wenig demokratischen Träumen einer staatsherrschaftlichen (Bildungs-)Politik des 19. Jahrhunderts als zu einer Bildungspolitik in einer von sehr unterschiedlichen Interessen geprägten, pluralistischen und zunehmend international verflochtenen liberalen Gesellschaft im 21. Jahrhundert.

\section{Anmerkungen}

1 Im Folgenden wird der Einfachheit halber der Begriff Bildungspolitik verwendet, obwohl ein Teil der von Walter Herzog kritisierten Reformprojekte nicht in den Parlamenten, sondern von den Bildungsverwaltungen initiiert worden ist. Systematisch müsste natürlich zwischen Bildungspolitik und Bildungsverwaltung unterschieden werden.

2 Auf die Berufsbildung, wo sich die Situation zum Teil anders präsentiert, wird an dieser Stelle nicht eingegangen (Späni, in Druck [2008]). 
3 Ob man dann auf eine evidenz-basierte Politik setzt, wie sie neuerdings die OECD für den Bildungsbereich gefordert hat (OECD/CERI, 2007a), oder eher der alten Rhetorik der Planung folgt (OECD, 1966), ändert nichts Grundlegendes. In beiden Fällen stellen sich letztlich die gleichen Grundfragen: Wer produziert wann zu welchem Zweck in wessen Auftrag welche Daten bzw. Forschungsergebnisse, wem gehören diese Daten bzw. Forschungsergebnisse und - damit verbunden - wer darf sie zu welchen Zwecken wie nutzen?

\section{Literatur}

Arnet, M. (2000). Das Schulkonkordat vom 29. Oktober 1970. Entstehung, Geschichte, Kommentar. Bern: EDK.

Bain, D., Brun, J., Hexel, D. \& Weiss, J. (2001). L’épopée des centres de recherche en éducation en Suisse 1960-2000 - Die Geschichte der Bildungsforschungsstellen in der Schweiz 19602000. Neuchâtel: IRDP.

Beck, U. \& Bonss, W. (1984). Soziologie und Modernisierung. Soziale Welt, 35 (4), 381-406.

Bourdieu, P. (1998). Vom Gebrauch der Wissenschaft. Für eine klinische Soziologie des wissenschaftlichen Feldes (S. 17). Konstanz: UVK.

Bundesrat (2005). Parlamentarische Initiative Bildungsrahmenartikel in der Bundesverfassung. Bericht vom 23. Juni 2005 der Kommission für Wissenschaft, Bildung und Kultur des Nationalrates. Stellungnahme des Bundesrates. Bundesblatt, 157, 5547-5556.

Caruso, M. \& Tenorth, H.-E. (Hrsg.). (2002). Internationalisierung. Semantik und Bildungssystem in vergleichender Perspektive. Bern: Lang.

Criblez, L. (1996). Schulreform durch New Public Management? Schweizer Schule (11), 322.

Criblez, L. (1999a). Entre profession et discipline: à propos du statut des sciences de l'éducation en Suisse. In R. Hofstetter \& B. Schneuwly, Le pari des sciences de l'éducation (pp. 169201). Paris/Bruxelles: De Boeck.

Criblez, L. (1999b). Requirements for a democratic education organization. Studies in philosophy and education, 18, 107-119.

Criblez, L. (2007). Bildungsföderalismus und Schulkoordination: konfligierende Prinzipien der Schulpolitik in der Schweiz. In R. Casale \& R. Horlacher (Hrsg.), Bildung und Öffentlichkeit. Jürgen Oelkers zum 60. Geburtstag (S. 262-276). Weinheim \& Basel: Beltz.

Criblez, L. (in Druck, 2008a). Die Reform der Lehrerinnen- und Lehrerbildung in der Schweiz seit 1990. Reformprozesse, erste Bilanz und Desiderata. In EDK (Hrsg.), Tertiarisierung der Lehrerinnen- und Lehrerbildung. Bilanz und Perspektiven. Bern: EDK.

Criblez, L. (in Druck, 2008b). Koordination und Harmonisierung. Die neue Bildungsverfassung und die Schulkoordination der Kantone. In L. Criblez (Hrsg.), Bildungsraum Schweiz - Geschichte und aktuelle Problemlagen. Bern: Haupt.

Crotti, C. (in Druck, 2008). Pädagogische Rekrutenprüfungen. Bildungspolitische Steuerungsversuche zwischen 1875 und 1931. In L. Criblez (Hrsg.), Bildungsraum SchweizGeschichte und aktuelle Problemlagen. Bern: Haupt.

Drerup, H. (1989). Probleme ausserwissenschaftlicher Verwendbarkeit von Erziehungswissenschaft. Zum Einfluss von Erziehungswissenschaft im politisch-administrativen Bereich. In E. König \& P. Zedler (Hrsg.), Rezeption und Verwendung erziehungswissenschaftlichen Wissens in pädagogischen Handlungs- und Entscheidungsfeldern (S. 143-165). Weinheim: Deutscher Studien Verlag.

Dubs, R. (1996). Schule, Schulentwicklung und New Public Management. St. Gallen: IWP.

EDK [Schweizerische Konferenz der kantonalen Erziehungsdirektoren] (1970). Konkordat über die Schulkoordination vom 29. Oktober 1970. In EDK (1986), Interkantonale Zusammenarbeit im Bildungswesen. Rechtsgrundlagen (S. 91-94). Bern: EDK.

EDK (1990). Bildungspolitik in der Schweiz. Bericht der OECD. Bern: EDK.

EDK (2004). HarmoS. Zielsetzungen und Konzeption. Bern: EDK. 
Gretler, A. (1982). Bemerkungen zum Selbstverständnis der Bildungsforschung in der Schweiz. Bildungsforschung und Bildungspraxis, 4, 117-123.

Hofstetter, R. (2007). Genève: de la pédagogie comme science morale aux sciences de l'éducation. Le déploiement d'un champ (pluri)disciplinaire (1890-1950). In R. Hofstetter \& B. Schneuwly (Hrsg.), Emergence des sciences de l'éducation en Suisse à la croisée de traditions académique contrastées (pp. 105-134). Bern: Lang.

Hofstetter, R. \& Schneuwly, B. (Hrsg.). (2007). Emergence des sciences de l'éducation en Suisse à la croisée de traditions académique contrastées. Bern: Lang.

Honegger, C., Jost, H.-U., Burren, S. \& Jurt, P. (2007). Konkurrierende Deutungen des Sozialen. Geschichts-, Sozial- und Wirtschaftswissenschaften im Spannungsfeld von Politik und Wissenschaft. Zürich: Chronos.

Kommission NR (2005). Kommission für Wissenschaft, Bildung und Kultur des Nationalrats. Parlamentarische Initiative Bildungsrahmenartikel in der Bundesverfassung. Bericht vom 23. Juni 2005. Bundesblatt, 157, 5479-5441.

Kussau, J. \& Oertel, L. (2001). Bildungsexpansion, Reform der Sekundarstufe I und Pädagogische Arbeitsstellen. Schweizerische Zeitschrift für Bildungswissenschaften, 23, 137-163.

Lehmann, L., Criblez, L., Guldimann, T., Fuchs, W. \& Périsset Bagnoud, D. (2007). Lehrerinnen- und Lehrerbildung in der Schweiz Aarau: SKBF.

Luhmann, N. (1987). Soziale Systeme. Grundriss einer allgemeinen Theorie. Frankfurt/M.: Suhrkamp.

Lustenberger, W. (1996). Pädagogische Rekrutenprüfungen. Ein Beitrag zur Schweizer Schulgeschichte. Chur/Zürich: Rüegger.

Maradan, O. \& Mangold, M. (2005). Bildungsstandards in der Schweiz: Das Projekt HarmoS. ph-akzente, 12 (2), 3-7.

Merton, R. K. (1936). The Unanticipated Consequences of Purposive Social Action. American Sociological Review, 1 (6), 894-904.

OECD (1966). Planification de l'enseignement. Problèmes d'organisation. Paris: OECD.

OECD/CERI (2007a). Evidence in Education. Linking Research and Policy. Paris: OECD.

OECD/CERI (2007b). National Review of Educational R\&D. Switzerland. Paris: OECD.

Osterwalder, F. \& Weber, K. (2004). Die Internationalisierung der föderalistischen Bildungspolitik. Schweizerische Zeitschrift für Bildungswissenschaften, 26, 11-32.

Rhyn, H. (1998). Schulaufsicht und Schulleitung in der deutschsprachigen Schweiz. Länderbericht Schweiz. In Bundesministerium für Unterricht und kulturelle Angelegenheiten Wien, Bund-Länder-Kommission für Bildungsplanung und Forschungsförderung Bonn \& Schweizerische Konferenz der kantonalen Erziehungsdirektoren Bern (Hrsg.), Schulleitung und Schulaufsicht. Neue Rollen und Aufgaben im Schulwesen einer dynamischen und offenen Gesellschaft (S. 163-187). Wien: Studien Verlag.

Rolff, H. G. (1984). Schule im Wandel. Kritische Analysen zur Schulentwicklung. Essen: Neue Deutsche Verlags-Gesellschaft.

Schedler, K. (1995). Ansätze einer wirkungsorientierten Verwaltungsführung. Bern: Haupt.

SKBF [Schweizerische Koordinationsstelle für Bildungsforschung] (2006). Bildungsbericht Schweiz 2006 (S. 7). Aarau: SKBF.

SKBF \& CORECHED [Schweizerische Koordinationsstelle für Bildungsforschung \& Conférence de coordination pour la recherche en éducation] (2006). Educational Research and Development in Switzerland. Country Background Report. Aarau: SKBF.

Späni, M. (in Druck, 2008). Der Bund und die Berufsbildung - von der «verfassungswidrigen Praxis» zum kooperativen Monopolisten. In L. Criblez (Hrsg.), Bildungsraum SchweizGeschichte und aktuelle Problemlagen. Bern: Haupt.

Stettler, N. (1994). «Die Zukunft ist errechenbar ...». In D. Blanc \& Ch. Luchsinger (Hrsg.), achtung: die 50er Jahre! (S. 95-117). Zürich: Chronos.

Stichweh, R. (2000). Die Weltgesellschaft. Frankfurt/M.: Suhrkamp. 
Vogel, C. (2006). Forschung und Entwicklung an den Pädagogischen Hochschulen und universitären Lehrerbildungsstätten der Schweiz. Stand und Entwicklungstendenzen 2005. Zürich: HfH.

Weingart, P. (2006). Erst denken, dann handeln? Wissenschaftliche Politikberatung aus der Perspektive der Wissens(chaft)soziologie. In S. Falk, D. Rehfeld, A. Römmele \& M. Thunert (Hrsg.), Handbuch Politikberatung (S. 35-44). Wiesbaden: VS Verlag für Sozialwissenschaften.

Widmaier, P. (1966). Bildungsplanung. Ansätze zu einer rationalen Bildungspolitik Stuttgart: Klett.

Widmer, K. (1976). Bildungsplanung und Schulreform. Frauenfeld/Stuttgart: Haupt.

Schlagworte: Bildungspolitik, Erziehungswissenschaft, Wissenschaftsforschung, educational policy 